TRANSACTIONS OF THE

AMERICAN MATHEMATICAL SOCIETY

Volume 363, Number 6, June 2011, Pages 3015-3030

S 0002-9947(2011)05278-5

Article electronically published on January 20, 2011

\title{
TOEPLITZ OPERATORS WITH BMO SYMBOLS ON THE SEGAL-BARGMANN SPACE
}

\author{
L. A. COBURN, J. ISRALOWITZ, AND BO LI
}

\begin{abstract}
We show that Zorboska's criterion for compactness of Toeplitz operators with $\mathrm{BMO}^{1}$ symbols on the Bergman space of the unit disc holds, by a different proof, for the Segal-Bargmann space of Gaussian square-integrable entire functions on $\mathbb{C}^{n}$. We establish some basic properties of $\mathrm{BMO}^{p}$ for $p \geq 1$ and complete the characterization of bounded and compact Toeplitz operators with $\mathrm{BMO}^{1}$ symbols. Via the Bargmann isometry and results of Lo and Engliš, we also give a compactness criterion for the Gabor-Daubechies "windowed Fourier localization operators" on $L^{2}\left(\mathbb{R}^{n}, d v\right)$ when the symbol is in a $\mathrm{BMO}^{1}$ Sobolev-type space. Finally, we discuss examples of the compactness criterion and counterexamples to the unrestricted application of this criterion for the compactness of Toeplitz operators.
\end{abstract}

\section{INTRODUCTION}

The Segal-Bargmann Hilbert space $H^{2}\left(\mathbb{C}^{n}, d \mu\right)$ of Gaussian square-integrable entire functions on complex $n$-space $[\mathrm{Ba}$ ] has the Bergman "reproducing kernel" property that

$$
f(z)=\int_{\mathbb{C}^{n}} K(z, w) f(w) d \mu(w)=\langle f(\cdot), K(\cdot, z)\rangle
$$

for all $f \in H^{2}\left(\mathbb{C}^{n}, d \mu\right)$ and $z \in \mathbb{C}^{n}$, where $z \cdot w=z_{1} \bar{w}_{1}+\cdots+z_{n} \bar{w}_{n},|z|^{2}=z \cdot z$, $K(z, w)=e^{\frac{z \cdot w}{2}}$ is the Bergman kernel function and

$$
d \mu(w)=(2 \pi)^{-n} e^{-\frac{|w|^{2}}{2}} d v(w),
$$

where $d v$ is Lebesgue volume measure. It is then easy to check that $k_{w}(z)=$ $K(z, w)\{K(w, w)\}^{-\frac{1}{2}}$ is a unit vector in the Hilbert space structure that $H^{2}\left(\mathbb{C}^{n}, d \mu\right)$ inherits as a subspace of $L^{2}\left(\mathbb{C}^{n}, d \mu\right)$. We sometimes denote $K(z, w)$ by $K_{w}(z)$.

We also consider the analogous Bergman spaces of square-integrable analytic functions $L_{a}^{2}(\Omega, d v)$ for $\Omega$ a bounded domain in $\mathbb{C}^{n}$ and the Gaussian $d \mu$ replaced by $d v$. Their kernel functions will also be denoted by $K(z, w)$ as in [K, pp. 39-54]. Note that $K(z, w)$ is always analytic in $z$ and conjugate-analytic in $w$ with

$$
\overline{K(z, w)}=K(w, z)
$$

and that $K(z, z)$ is positive. The "bounded symmetric domains" $\Omega$ give interesting special cases which are "closest" to the model $H^{2}\left(\mathbb{C}^{n}, d \mu\right)$ BBCZ.

Received by the editors September 24, 2008 and, in revised form, March 2, 2009.

2010 Mathematics Subject Classification. Primary 47B32; Secondary 32A36.

(C)2011 American Mathematical Society 
Let $z \in \mathbb{C}^{n}$ and let $d \nu_{z}$ denote the probability measure $d \nu_{z}(w)=\left|k_{z}(w)\right|^{2} d \mu(w)$. For $1 \leq p<\infty$, let $\mathcal{T}^{p}\left(\mathbb{C}^{n}\right)$ be the set of all measurable $f$ such that for every $z \in \mathbb{C}^{n}, f \in L^{p}\left(\mathbb{C}^{n}, d \nu_{z}\right)$. If $p=2$, then we will write $\mathcal{T}\left(\mathbb{C}^{n}\right)=\mathcal{T}^{2}\left(\mathbb{C}^{n}\right)$.

Let $P$ denote the integral operator on $\mathcal{T}^{1}\left(\mathbb{C}^{n}\right)$ given by

$$
(P g)(z)=\int_{\mathbb{C}^{n}} g(w) K(z, w) d \mu(w) .
$$

Note that for arbitrary $g$ in $L^{2}\left(\mathbb{C}^{n}, d \mu\right), P g$ is the orthogonal projection of $g$ onto $H^{2}\left(\mathbb{C}^{n}, d \mu\right)$.

For $f$ in $\mathcal{T}^{1}\left(\mathbb{C}^{n}\right)$ and $M_{f}$ the operator of "multiplication by $f$ " we define the Toeplitz operator $T_{f}$ to be the integral operator $T_{f}=P M_{f} P$ on $L^{2}\left(\mathbb{C}^{n}, d \mu\right)$. This operator is densely defined when $f$ is in $\mathcal{T}\left(\mathbb{C}^{n}\right)$ and is known to be bounded from $L^{2}\left(\mathbb{C}^{n}, d \mu\right)$ to $L^{2}\left(\mathbb{C}^{n}, d \mu\right)$ for special choices of $f$ in $\mathcal{T}^{1}\left(\mathbb{C}^{n}\right)$ [Bau, [BC1, [BC2]. In particular, for bounded $f$, the boundedness of $T_{f}$ is obvious.

For the Bergman spaces $L_{a}^{2}(\Omega)$ and for $H^{2}\left(\mathbb{C}^{n}, d \mu\right)$, the Toeplitz operators $T_{f}$ with "symbol" $f$ have been extensively studied [AZ, $\mathrm{BC1},[\mathrm{BC} 2,[\mathrm{E} 1,[\mathrm{~F},[\mathrm{Z}]$. We will give a brief discussion of these operators for $H^{2}\left(\mathbb{C}^{n}, d \mu\right)$ : the situation for $L_{a}^{2}(\Omega)$ is completely analogous. For $f$ in $\mathcal{T}\left(\mathbb{C}^{n}\right)$, the Toeplitz operator $T_{f}$ is defined by $T_{f} h=P(f h)$ on a dense subspace of $H^{2}\left(\mathbb{C}^{n}, d \mu\right)$. The Berezin transform $\mathrm{Be}_{2}$ of $T_{f}$ is the function on $\mathbb{C}^{n}$ defined by

$$
\widetilde{T}_{f}(z)=\left\langle T_{f} k_{z}, k_{z}\right\rangle .
$$

It is a "smoothed" version of the symbol $f$. For $f \in \mathcal{T}^{p}\left(\mathbb{C}^{n}\right)$, we define the Berezin transform of $f$ by $\tilde{f}(z)=\int_{\mathbb{C}^{n}} f(w)\left|k_{z}(w)\right|^{2} d \mu(w)$. Note that by the definition of $T_{f}$, we have $\tilde{f}=\widetilde{T_{f}}$ for $f \in \mathcal{T}\left(\mathbb{C}^{n}\right)$.

For $\mathbb{C}^{n}$ or for the bounded symmetric domains $\Omega$ in $\mathbb{C}^{n}$, there are well-known families of involutive automorphisms $\tau_{z}(\cdot)$ for all $z$ in the domain. For $\mathbb{C}^{n}, \tau_{z}(u)=$ $z-u$ while for $\Omega=\mathbb{D}$, the open unit disc in $\mathbb{C}, \tau_{z}(u)=(z-u)(1-\bar{z} u)^{-1}$. Using the $\tau_{z}$, we follow Zorboska [Z] and say that $f$ is in $\operatorname{BMO}^{1}\left(\mathbb{C}^{n}\right)$ if $f \in \mathcal{T}^{1}\left(\mathbb{C}^{n}\right)$ and

$$
\sup _{z \in \mathbb{C}^{n}}\left\|f \circ \tau_{z}-\tilde{f}(z)\right\|_{L^{1}\left(\mathbb{C}^{n}, d \mu\right)}<\infty .
$$

For a function $f \in \mathrm{BMO}^{1}\left(\mathbb{C}^{n}\right)$, we denote the supremum above by $\|f\|_{\mathrm{BMO}^{1}\left(\mathbb{C}^{n}\right)}$. Note that for $f$ in $\mathcal{T}^{p}\left(\mathbb{C}^{n}\right)$,

$$
\tilde{f}(z)=\int_{\mathbb{C}^{n}} f \circ \tau_{z}(u) d \mu(u) .
$$

It is then easy to see that for each $z \in \mathbb{C}^{n}, \widetilde{f \circ \tau_{z}}=\tilde{f} \circ \tau_{z}$ and $\|f\|_{\mathrm{BMO}^{1}\left(\mathbb{C}^{n}\right)}=$ $\left\|f \circ \tau_{z}\right\|_{\mathrm{BMO}^{1}\left(\mathbb{C}^{n}\right)}$.

If $f \in \mathcal{T}^{p}\left(\mathbb{C}^{n}\right)$ for $1 \leq p<2$, then it is not clear if the Toeplitz operator $T_{f}$ is densely defined. In section 3 , we will show that $T_{f}$ is densely defined and $\tilde{f}=\widetilde{T_{f}}$ when $f$ is in $\mathrm{BMO}^{1}\left(\mathbb{C}^{n}\right)$. In Theorem 2, we will show that $T_{f}$ is well defined and bounded when $f$ is in $\operatorname{BMO}^{1}\left(\mathbb{C}^{n}\right)$ and $\tilde{f}$ is bounded.

When $\Omega=\mathbb{D}$ is the open unit disc in $\mathbb{C}$ and $f$ is bounded, $\left[\mathrm{AZ}\right.$ showed that $T_{f}$ is compact if and only if

$$
\lim _{|z| \rightarrow 1} \tilde{f}(z)=0
$$


The corresponding result was obtained by $\left[\mathrm{E} 1\right.$ for bounded $f$, where $L_{a}^{2}(\mathbb{D})$ is replaced by the Segal-Bargmann space $H^{2}\left(\mathbb{C}^{n}, d \mu\right)$. Here, $T_{f}$ is compact if and only if

$$
\lim _{|z| \rightarrow+\infty} \tilde{f}(z)=0 .
$$

The result of $\left[\mathrm{AZ}\right.$ has been shown by Zorboska $\mathrm{Z}$ to hold for all $f$ in $\mathrm{BMO}^{1}(\mathbb{D})$. We show, by different methods, that the result of $\left[\mathrm{Z}\right.$ holds for $H^{2}\left(\mathbb{C}^{n}, d \mu\right)$ and $f \in \mathrm{BMO}^{1}\left(\mathbb{C}^{n}\right)$, extending the results of [E1]. As pointed out in [E1], examples in $\left[\mathrm{BC} 2\right.$, p. 582] show that some restriction, such as $\mathrm{BMO}^{1}\left(\mathbb{C}^{n}\right)$, is needed for this result to hold. For related results, see also $[\mathrm{BF}$ and $[\mathrm{MZ}$.

Using the Bargmann isometry $\beta: L^{2}\left(\mathbb{R}^{n}, d v\right) \rightarrow H^{2}\left(\mathbb{C}^{n}, d \mu\right)$ Ba and results of Lo $[\mathrm{L}$ and Englis $[\mathrm{E} 2$, our result provides a compactness criterion for the GaborDaubechies "windowed Fourier localization operators" $[\mathrm{D}] L_{f}^{(w)}$ on $L^{2}\left(\mathbb{R}^{n}, d v\right)$, where the "window" $w$ is a linear combination of Hermite functions, the "symbol" $f$ is in an appropriate $\mathrm{BMO}^{1}$ Sobolev-type space, and the operator $L_{f}^{(w)}$ is defined by

$$
\left\langle L_{f}^{(w)} g, h\right\rangle=(2 \pi)^{-n} \int_{\mathbb{C}^{n}} f(z)\left\langle\beta g, W_{z} \beta w\right\rangle\left\langle W_{z} \beta w, \beta h\right\rangle d v(z) .
$$

Here, $W_{z}$ is the Weyl operator on $H^{2}\left(\mathbb{C}^{n}, d \mu\right)$ given by

$$
W_{z} u(a)=e^{\frac{a \cdot z}{2}-\frac{|z|^{2}}{4}} u(a-z) .
$$

In the next section, we set up some machinery and prove the main result.

\section{MAIN RESUlt}

As mentioned in the introduction above, our main result is the following:

Theorem 1. Let $f \in B M O^{1}\left(\mathbb{C}^{n}\right)$. Then $\lim _{|z| \rightarrow+\infty} \tilde{f}(z)=0$ implies that the Toeplitz operator $T_{f}$ is compact on $H^{2}\left(\mathbb{C}^{n}, d \mu\right)$.

Similar to the method of proof in [AZ] and [Z, we will ultimately use the Schur test to prove our main result. However, to be able to use the Schur test, we will have to use methods that differ from the ones found in $[\mathrm{Z}]$ and $[\mathrm{AZ}]$.

Note that we have

$$
\left\|f \circ \tau_{z}-\tilde{f}(z)\right\|_{L^{1}\left(\mathbb{C}^{n}, d \mu\right)} \leq\left\|f \circ \tau_{z}\right\|_{L^{1}\left(\mathbb{C}^{n}, d \mu\right)}+|\tilde{f}(z)| \leq 2 \widetilde{|f|}(z) .
$$

It is easy to check that the space $\mathrm{BMO}^{2}\left(\mathbb{C}^{n}\right)$ of $\mathrm{Bau}$ is contained in $\mathrm{BMO}^{1}\left(\mathbb{C}^{n}\right)$. Moreover, using

$$
\tilde{f}(z)=(2 \pi)^{-n} \int_{\mathbb{C}^{n}} f(w) e^{-\frac{|z-w|^{2}}{2}} d v(w),
$$

we see that, for any $f=g+b$ with $g \in L^{p}\left(\mathbb{C}^{n}, d v\right)$ for some $p \geq 1$ and $b$ essentially bounded, an easy application of Hölder's inequality shows that $f \in \mathrm{BMO}^{1}\left(\mathbb{C}^{n}\right)$ with $\widetilde{|f|}$ bounded. Thus, our main result gives a nontrivial extension of the corresponding result in [E1].

We now have

Lemma 1. If $f \in B M O^{1}\left(\mathbb{C}^{n}\right)$, then $\sup _{z \in \mathbb{C}^{n}}(\widetilde{|f|}(z)-|\tilde{f}(z)|)<\infty$. 
Proof. By definition, and using the fact that each $k_{z}$ is a unit vector in $H^{2}\left(\mathbb{C}^{n}, d \mu\right)$, we have

$$
\begin{aligned}
\widetilde{|f|}|(z)-| \tilde{f}(z) \mid & =\int_{\mathbb{C}^{n}}(|f(w)|-|\tilde{f}(z)|)\left|k_{z}(w)\right|^{2} d \mu(w) \\
& \leq \int_{\mathbb{C}^{n}}|f(w)-\tilde{f}(z)|\left|k_{z}(w)\right|^{2} d \mu(w) \\
& =\int_{\mathbb{C}^{n}}\left|f \circ \tau_{z}(w)-\tilde{f}(z)\right| d \mu(w),
\end{aligned}
$$

where in the last equation we used the change of variables $w \mapsto \tau_{z}(w)$. The lemma then follows from the definition of $\mathrm{BMO}^{1}\left(\mathbb{C}^{n}\right)$.

Corollary. If $f \in B M O^{1}\left(\mathbb{C}^{n}\right)$ and $\sup _{z \in \mathbb{C}^{n}}|\tilde{f}(z)|<\infty$, then $\sup _{z \in \mathbb{C}^{n}} \widetilde{|f|}(z) \leq$ $\|f\|_{B M O^{1}\left(\mathbb{C}^{n}\right)}+\sup _{z \in \mathbb{C}^{n}}|\tilde{f}(z)|$.

Proof. Since $\widetilde{|f|}|(z)=(\widetilde{|f|}(z)-|\tilde{f}(z)|)+| \tilde{f}(z) \mid$ the result follows from the previous lemma.

Remark. If $f \in \mathrm{BMO}^{1}\left(\mathbb{C}^{n}\right)$ and $\sup _{z \in \mathbb{C}^{n}}|\tilde{f}(z)|<\infty$, then for any $s \in \mathbb{C}^{n}$, an easy calculation gives us that

$$
\sup _{z \in \mathbb{C}^{n}}|\tilde{f}(z)|+\|f\|_{\mathrm{BMO}^{1}\left(\mathbb{C}^{n}\right)}=\sup _{z \in \mathbb{C}^{n}}\left|\widetilde{f \circ \tau_{s}}(z)\right|+\left\|f \circ \tau_{s}\right\|_{\mathrm{BMO}^{1}\left(\mathbb{C}^{n}\right)} .
$$

To simplify notation in the sequel, we will sometimes let $L^{2}$ denote $L^{2}\left(\mathbb{C}^{n}, d \mu\right)$ and let $H^{2}$ denote $H^{2}\left(\mathbb{C}^{n}, d \mu\right)$.

Proposition 1. If $f \in B M O^{1}\left(\mathbb{C}^{n}\right)$ and $\tilde{f}$ is bounded, then $T_{|f|}=P M_{|f|} P$ is bounded on $L^{2}$ with

$$
\left\|T_{|f|}\right\|_{L^{2} \rightarrow L^{2}} \leq 4^{n}\left(\|f\|_{B M O^{1}\left(\mathbb{C}^{n}\right)}+\sup _{z \in \mathbb{C}^{n}}|\tilde{f}(z)|\right)
$$

and the closed densely defined operator $M_{|f|^{\frac{1}{2}}} P$ is bounded on $L^{2}$ with

$$
\left\|M_{|f|^{\frac{1}{2}}} P\right\|_{L^{2} \rightarrow L^{2}}^{2} \leq\left\|T_{|f|}\right\|_{L^{2} \rightarrow L^{2}}
$$

Here, $M_{g}$ is the operator of "multiplication by $g$ ".

Proof. By the corollary to Lemma 1,

$$
\sup _{z \in \mathbb{C}^{n}} \widetilde{|f|}(z) \leq\|f\|_{\mathrm{BMO}^{1}\left(\mathbb{C}^{n}\right)}+\sup _{z \in \mathbb{C}^{n}}|\tilde{f}(z)| .
$$

From [BC1, p. 823] and [Bau, p. 7], we have $\left\|T_{|f|}\right\|_{L^{2} \rightarrow L^{2}} \leq 4^{n}\left(\sup _{z \in \mathbb{C}^{n}} \widetilde{|f|}(z)\right)$. For $A=M_{|f|^{\frac{1}{2}}} P$, by $\left[\underline{\mathrm{RN}}\right.$, pp. 297-300], $A^{*}$ is an extension of $P M_{|f|^{\frac{1}{2}}}$. Note that since $L^{2}=H^{2} \oplus\left(H^{2}\right)^{\perp}$ and the linear span of $\left\{K_{z}: z \in \mathbb{C}^{n}\right\}$ is dense in $H^{2}$, it is easy to see that $A$ has dense domain. Since $\left(P M_{|f|^{\frac{1}{2}}}\right)\left(M_{|f|^{\frac{1}{2}}} P\right)=T_{|f|}$ is a bounded operator, for $h$ in the (dense) domain of $A$,

$$
A^{*}(A h)=T_{|f|} h
$$

and so

$$
\langle A h, A h\rangle=\left\langle h, A^{*}(A h)\right\rangle=\left\langle h, T_{|f|} h\right\rangle .
$$


It follows, by the Cauchy-Schwarz inequality, that

$$
\left\|M_{|f|^{\frac{1}{2}}} P\right\|_{L^{2} \rightarrow L^{2}}^{2} \leq\left\|T_{|f|}\right\|_{L^{2} \rightarrow L^{2}}
$$

Now, we have

Theorem 2. If $f \in B M O^{1}\left(\mathbb{C}^{n}\right)$ and $\tilde{f}$ is bounded, then $T_{f}$ is well defined and bounded on $H^{2}\left(\mathbb{C}^{n}, d \mu\right)$.

Proof. Because $f$ is in $\mathrm{BMO}^{1}\left(\mathbb{C}^{n}\right)$ and $\tilde{f}$ is bounded, Lemma 1 shows that $\widetilde{|f|}$ is bounded. Consider $g \equiv(\operatorname{Re} f)^{+}$, the "positive part" of Re $f$. We see that $g$ is integrable with $0 \leq g \leq|f|$. It follows that $\tilde{g}$ is bounded and so $g$ is in $\operatorname{BMO}^{1}\left(\mathbb{C}^{n}\right)$. The argument of Proposition 1 now shows that $T_{g}$ is bounded. It follows easily that $T_{f}$ is bounded.

Remark 1. For $f \in \mathrm{BMO}^{1}\left(\mathbb{C}^{n}\right)$ with $\tilde{f}$ bounded, $T_{f}=P M_{f} P$ is defined as an integral operator. By Theorem 2, $T_{f}$ is bounded. It is not obvious, however, that $T_{f}: L^{2} \rightarrow H^{2}$, or that $T_{f}^{*}=T_{\bar{f}}$ on $L^{2}$ and $\widetilde{T_{f}}=\tilde{f}$. These assertions hold, but must be checked by Fubini's theorem. That is, one must use Fubini's theorem to check that $\left\langle T_{f} K_{b}, K_{a}\right\rangle=\int_{\mathbb{C}^{n}} f(w) K_{b}(w) \overline{K_{a}(w)} d \mu(w)$ for each $a$ and $b$ in $\mathbb{C}^{n}$. In particular,

$$
\begin{aligned}
& \int_{\mathbb{C}^{n}}\left(\int_{\mathbb{C}^{n}}\left|f(w) K_{a}(w) e^{\frac{u \cdot w}{2}}\right| d \mu(w)\right)\left|K_{b}(u)\right| d \mu(u) \\
& \leq \int_{\mathbb{C}^{n}}\left[\left(\int_{\mathbb{C}^{n}}\left(|f(w)|^{\frac{1}{2}}\left|K_{a}(w)\right|\right)^{2} d \mu(w)\right)^{\frac{1}{2}}\right. \\
& \left.\quad \times\left(\int_{\mathbb{C}^{n}}\left(|f(w)|^{\frac{1}{2}}\left|e^{\frac{u \cdot w}{2}}\right|\right)^{2} d \mu(w)\right)^{\frac{1}{2}}\right]\left|K_{b}(u)\right| d \mu(u) \\
& \leq e^{\frac{|a|^{2}}{4}}\left\|M_{|f|^{\frac{1}{2}}}\right\|_{H^{2} \rightarrow L^{2}}^{2} \int_{\mathbb{C}^{n}}\left|K_{b}(u)\right| e^{\frac{|u|^{2}}{4}} d \mu(u) \\
& <\infty .
\end{aligned}
$$

Note that the proof of Theorem 3 will also tell us that the integral in (1) is finite. The equation $P T_{f} K_{a}=T_{f} K_{a}$, which implies that $T_{f}: L^{2} \rightarrow H^{2}$, now follows. Similarly, the equations $T_{f}^{*}=T_{\bar{f}}$ on $L^{2}$ and $\widetilde{T_{f}}=\tilde{f}$ follow easily, using the boundedness of $T_{f}$. As stated in the introduction, we will show in section 3 that $T_{f}$ maps a dense linear subspace of $L^{2}$ into $H^{2}$ and show that $\widetilde{T_{f}}=\tilde{f}$ when $f$ is in $\operatorname{BMO}^{1}\left(\mathbb{C}^{n}\right)$. In section 3 we will also prove the fact, used implicitly in the proof of Theorem 1 , that $\tilde{f}$ is continuous for all $f \in \mathrm{BMO}^{1}\left(\mathbb{C}^{n}\right)$.

Corollary. For $f \in B M O^{1}\left(\mathbb{C}^{n}\right)$ with $\tilde{f}$ bounded, we have that $\tilde{f}$ is Lipschitz.

Proof. This follows immediately from [C, Theorem 2].

Remark 2. The above corollary is an improvement of [BC1, Lemma 2]. We will further discuss the issue of $\tilde{f}$ being Lipschitz in section 3 .

We will proceed with the proof of our main result in a step-by-step manner. First, we need a preliminary identity. 
Lemma 2. If $f \in B M O^{1}\left(\mathbb{C}^{n}\right)$ with $\tilde{f}$ bounded, then

$$
\left(T_{f} K_{z}\right)(u)=K_{z}(u)\left[P\left(f \circ \tau_{z}\right)\right]\left(\tau_{z}(u)\right) .
$$

Proof. From the hypothesis, $f \circ \tau_{z} \in \mathrm{BMO}^{1}\left(\mathbb{C}^{n}\right)$ with $\widetilde{f \circ \tau_{z}}$ bounded for each $z \in \mathbb{C}^{n}$, so that each side of the equation in Lemma 2 is well defined. By definition,

$$
\begin{aligned}
T_{f} K_{z}(u) & =P\left(f K_{z}\right)(u) \\
& =\int_{\mathbb{C}^{n}} f(w) K_{z}(w) \overline{K_{u}(w)} d \mu(w) \\
& =\int_{\mathbb{C}^{n}} f\left(\tau_{z}(w)\right) K_{z}\left(\tau_{z}(w)\right) \overline{K_{u}\left(\tau_{z}(w)\right)}\left|k_{z}(w)\right|^{2} d \mu(w),
\end{aligned}
$$

where we get the last equality by the change of variables $w \mapsto \tau_{z}(w)$.

Moreover,

$$
\begin{aligned}
K_{z}\left(\tau_{z}(w)\right) \overline{K_{u}\left(\tau_{z}(w)\right)}\left|k_{z}(w)\right|^{2} & =e^{\frac{(z-w) \cdot z}{2}} e^{\frac{u \cdot(z-w)}{2}} e^{\frac{w \cdot z}{2}} e^{\frac{z \cdot w}{2}} e^{-\frac{|z|^{2}}{2}} \\
& =e^{\frac{u \cdot z}{2}-\frac{u \cdot w}{2}+\frac{z \cdot w}{2}}
\end{aligned}
$$

However,

$$
\begin{aligned}
K_{z}(u) P\left(f \circ \tau_{z}\right)\left(\tau_{z}(u)\right) & =e^{\frac{u \cdot z}{2}} \int_{\mathbb{C}^{n}} f\left(\tau_{z}(w)\right) e^{\frac{(z-u) \cdot w}{2}} d \mu(w) \\
& =\int_{\mathbb{C}^{n}} f\left(\tau_{z}(w)\right) e^{\frac{u \cdot z}{2}-\frac{u \cdot w}{2}+\frac{z \cdot w}{2}} d \mu(w),
\end{aligned}
$$

which proves the lemma.

Proof of Theorem 1.

Step 1. For $f \in \operatorname{BMO}^{1}\left(\mathbb{C}^{n}\right)$ and $\tilde{f}$ bounded, we have that

$$
\sup _{z \in \mathbb{C}^{n}}\left|P\left(f \circ \tau_{z}\right)(w)\right| \leq K_{f} e^{\frac{|w|^{2}}{8}}
$$

for some $K_{f}>0$ only depending on $f$.

Proof. Let $K_{f}^{\prime}=\sup _{z \in \mathbb{C}^{n}}|\tilde{f}(z)|+\|f\|_{\mathrm{BMO}^{1}\left(\mathbb{C}^{n}\right)}$. For any $z \in \mathbb{C}^{n}$, Proposition 1 and the remark following the corollary to Lemma 1 give us that

$$
\left.M_{\left|f \circ \tau_{z}\right|^{\frac{1}{2}}} P\right|_{H^{2}}=M_{\left|f \circ \tau_{z}\right|^{\frac{1}{2}}}
$$

is bounded on $H^{2}\left(\mathbb{C}^{n}, d \mu\right)$ with $\left\|M_{\left|f \circ \tau_{z}\right|^{\frac{1}{2}}}\right\|_{H^{2} \rightarrow L^{2}}^{2} \leq 4^{n} K_{f}^{\prime}$. Thus, as $e^{\frac{\sigma \cdot w}{4}}$ is in $H^{2}$ for each $w \in \mathbb{C}^{n}$, we have that

$$
\begin{aligned}
\left|P\left(f \circ \tau_{z}\right)(w)\right| & \leq\left.\int_{\mathbb{C}^{n}}\left|\left(f \circ \tau_{z}\right)(\sigma)\right|^{\frac{1}{2}} e^{\frac{\sigma \cdot w}{4}}\right|^{2} d \mu(\sigma) \\
& \leq 4^{n} K_{f}^{\prime} \int_{\mathbb{C}^{n}}\left|e^{\frac{\sigma \cdot w}{2}}\right| d \mu(\sigma) .
\end{aligned}
$$

However, it is elementary that

$$
\int_{\mathbb{C}^{n}}\left|e^{\frac{\sigma \cdot w}{2}}\right| d \mu(\sigma)=e^{\frac{|w|^{2}}{8}}
$$

which proves Step 1 with $K_{f}=4^{n} K_{f}^{\prime}$. 
Step 2. By Theorem 2, $T_{f}$ is bounded on $H^{2}\left(\mathbb{C}^{n}, d \mu\right)$ if $f \in \operatorname{BMO}^{1}\left(\mathbb{C}^{n}\right)$ and $\tilde{f}$ is bounded. For $\tilde{f}(z) \rightarrow 0$ as $|z| \rightarrow+\infty$, we have $T_{f \circ \tau_{z}} \mathbf{1} \rightarrow 0$ weakly as $|z| \rightarrow \infty$. In particular, $\left(T_{f \circ \tau_{z}} \mathbf{1}\right)(w)=\left\langle T_{f \circ \tau_{z}} \mathbf{1}, K(\cdot, w)\right\rangle \rightarrow 0$ pointwise as $|z| \rightarrow+\infty$.

Proof. The proof is similar to the corresponding result in [AZ, where the only major difference is that the multinomial theorem is used to obtain the double series formula for $\tilde{S}$, where $S$ is any bounded operator on $H^{2}\left(\mathbb{C}^{n}, d \mu\right)$ and $\tilde{S}(z)=\left\langle S k_{z}, k_{z}\right\rangle$. We leave the details to the interested reader.

Step 3. Let $f \in \operatorname{BMO}^{1}\left(\mathbb{C}^{n}\right)$ and $\tilde{f}(z) \rightarrow 0$ as $|z| \rightarrow+\infty$. For $R>0$, let $B(0, R)$ denote the open Euclidean ball of radius $R$ centered at 0 . Let $T_{R}^{f}$ denote the operator from $H^{2}\left(\mathbb{C}^{n}, d \mu\right)$ into $L^{2}\left(\mathbb{C}^{n}, d \mu\right)$ defined by

$$
T_{R}^{f}=M_{\chi_{B(0, R)}} T_{f},
$$

where $M_{\chi_{B(0, R)}}$ is the multiplication operator with respect to the characteristic function $\chi_{B(0, R)}(\cdot)$ on $B(0, R)$. Let $T^{f}$ denote the operator $T_{f}$ as an operator from $H^{2}\left(\mathbb{C}^{n}, d \mu\right)$ into $L^{2}\left(\mathbb{C}^{n}, d \mu\right)$. Then $T_{R}^{f}$ is compact and $\lim _{R \rightarrow+\infty}\left\|T^{f}-T_{R}^{f}\right\|_{H^{2} \rightarrow L^{2}}=$ 0 , so that $T_{f}: H^{2}\left(\mathbb{C}^{n}, d \mu\right) \rightarrow H^{2}\left(\mathbb{C}^{n}, d \mu\right)$ is compact.

Proof. By Theorem 2, $T_{f}$ is bounded. By a simple normal family argument, $M_{\chi_{B(0, R)}}: H^{2}\left(\mathbb{C}^{n}, d \mu\right) \rightarrow L^{2}\left(\mathbb{C}^{n}, d \mu\right)$ is compact, and so each $T_{R}^{f}$ is compact. For $g \in H^{2}\left(\mathbb{C}^{n}, d \mu\right)$, we have that

$$
\begin{aligned}
\left(T^{f}-T_{R}^{f}\right) g(z) & =\left(\left(1-\chi_{B(0, R)}\right) T_{f} g\right)(z) \\
& =\left(1-\chi_{B(0, R)}(z)\right)\left\langle T_{f} g, K_{z}\right\rangle=\left(1-\chi_{B(0, R)}(z)\right)\left\langle g, T_{\bar{f}} K_{z}\right\rangle \\
& =\int_{\mathbb{C}^{n}} g(u)\left(1-\chi_{B(0, R)}(z)\right) \overline{T_{\bar{f}} K_{z}}(u) d \mu(u)
\end{aligned}
$$

so that $T^{f}-T_{R}^{f}$ is an integral operator on $H^{2}\left(\mathbb{C}^{n}, d \mu\right)$ with kernel $K_{R}^{f}(z, u)=$ $\left(1-\chi_{B(0, R)}(z)\right) \overline{T_{\bar{f}} K_{z}}(u)$. By Schur's test [HS, p. 22], whenever there exists a positive measurable function $h$ on $\mathbb{C}^{n}$ and constants $C_{1, R}$ and $C_{2}$ such that

$$
\begin{gathered}
\int_{\mathbb{C}^{n}}\left|K_{R}^{f}(z, u)\right| h(z) d \mu(z) \leq C_{2} h(u), \quad \forall u \in \mathbb{C}^{n}, \\
\int_{\mathbb{C}^{n}}\left|K_{R}^{f}(z, u)\right| h(u) d \mu(u) \leq C_{1, R} h(z), \quad \forall z \in \mathbb{C}^{n},
\end{gathered}
$$

we have that $\left\|T^{f}-T_{R}^{f}\right\|_{H^{2} \rightarrow L^{2}}^{2} \leq C_{1, R} C_{2}$.

Let $h(z)=\left(K_{z}(z)\right)^{\frac{1}{2}}=e^{\frac{|z|^{2}}{4}}$, so that for $|z| \geq R$, we have

$$
\int_{\mathbb{C}^{n}}\left|K_{R}^{f}(z, u)\right| h(u) d \mu(u)=(2 \pi)^{-n} \int_{\mathbb{C}^{n}}\left|T_{\bar{f}} K_{z}(u)\right|\left(K_{u}(u)\right)^{\frac{1}{2}} e^{-\frac{|u|^{2}}{2}} d v(u),
$$

which by Lemma 2 is equal to

$$
(2 \pi)^{-n} \int_{\mathbb{C}^{n}}\left|K_{z}(u)\right|\left|P\left(\bar{f} \circ \tau_{z}\right)\left(\tau_{z}(u)\right)\right|\left(K_{u}(u)\right)^{\frac{1}{2}} e^{-\frac{|u|^{2}}{2}} d v(u) .
$$

By the change of variables $u \rightarrow \tau_{z}(u)$, this is equal to

$$
(2 \pi)^{-n} \int_{\mathbb{C}^{n}}\left|K_{z}\left(\tau_{z}(u)\right)\right|\left|P\left(\bar{f} \circ \tau_{z}\right)(u)\right|\left(K_{\tau_{z}(u)}\left(\tau_{z}(u)\right)\right)^{\frac{1}{2}} e^{-\frac{|z-u|^{2}}{2}} d v(u) .
$$


Since $\left|K_{z}\left(\tau_{z}(u)\right)\right|=\left|e^{\frac{(z-u) \cdot z}{2}}\right|$ and $\left(K_{\tau_{z}(u)}\left(\tau_{z}(u)\right)\right)^{\frac{1}{2}}=e^{\frac{|z-u|^{2}}{4}}$, this becomes

$$
(2 \pi)^{-n} \int_{\mathbb{C}^{n}}\left|P\left(\bar{f} \circ \tau_{z}\right)(u)\right|\left|e^{\frac{(z-u) \cdot z}{2}}\right| e^{-\frac{|z-u|^{2}}{4}} d v(u) .
$$

If $1<p<\infty$ with conjugate exponent $q$, then by Hölder's inequality, we get that

$$
\begin{aligned}
& (2 \pi)^{-n} \int_{\mathbb{C}^{n}}\left|P\left(\bar{f} \circ \tau_{z}\right)(u)\right|\left|e^{\frac{(z-u) \cdot z}{2}}\right| e^{-\frac{|z-u|^{2}}{4}} d v(u) \\
& =(2 \pi)^{-n} \int_{\mathbb{C}^{n}}\left(\left|P\left(\bar{f} \circ \tau_{z}\right)(u)\right| e^{-\frac{|u|^{2}}{5}}\right)\left(e^{\frac{|u|^{2}}{5}}\left|e^{\frac{(z-u) \cdot z}{2}}\right| e^{-\frac{|z-u|^{2}}{4}}\right) d v(u) \\
& \leq(2 \pi)^{-n}\left(\int_{\mathbb{C}^{n}}\left|P\left(\bar{f} \circ \tau_{z}\right)(u)\right|^{p} e^{-\frac{p|u|^{2}}{5}} d v(u)\right)^{\frac{1}{p}} \\
& \times\left(\int_{\mathbb{C}^{n}} e^{\frac{q|u|^{2}}{5}}\left|e^{\frac{q(z-u) \cdot z}{2}}\right| e^{-\frac{q|z-u|^{2}}{4}} d v(u)\right)^{\frac{1}{q}} .
\end{aligned}
$$

Let

$$
C_{1, R}^{\prime}=\sup _{|z| \geq R}\left(\int_{\mathbb{C}^{n}}\left|P\left(\bar{f} \circ \tau_{z}\right)(u)\right|^{p} e^{-\frac{p|u|^{2}}{5}} d v(u)\right)^{\frac{1}{p}} .
$$

From Step 1, we have that $\left|P\left(\bar{f} \circ \tau_{z}\right)(u)\right|^{p} e^{-\frac{p|u|^{2}}{5}} \leq K_{f}^{p} e^{-\frac{3 p|u|^{2}}{40}}$, and from Step 2, we have that $\lim _{|z| \rightarrow+\infty} P\left(\bar{f} \circ \tau_{z}\right)(u)=0$ pointwise in $u$. Thus, by the dominated convergence theorem, $C_{1, R}^{\prime} \rightarrow 0$ as $R \rightarrow+\infty$. Moreover,

$$
\begin{aligned}
e^{\frac{q|u|^{2}}{5}}\left|e^{\frac{q(z-u) \cdot z}{2}}\right| e^{-\frac{q|z-u|^{2}}{4}} & =e^{\frac{q|u|^{2}}{5}} e^{\frac{q|z|^{2}}{2}} e^{-\frac{q \operatorname{Re}\{u \cdot z\}}{2}} e^{-\frac{q|z|^{2}}{4}} e^{\frac{q \operatorname{Re}\{u \cdot z\}}{2}} e^{-\frac{q|u|^{2}}{4}} \\
& =e^{\frac{q|z|^{2}}{4}} e^{-\frac{q|u|^{2}}{20}}
\end{aligned}
$$

so that

$$
(2 \pi)^{-n}\left(\int_{\mathbb{C}^{n}} e^{\frac{q|u|^{2}}{5}}\left|e^{\frac{q(z-u) \cdot z}{2}}\right| e^{-\frac{q|z-u|^{2}}{4}} d v(u)\right)^{\frac{1}{q}}=(2 \pi)^{-n} e^{\frac{|z|^{2}}{4}}\left(\int_{\mathbb{C}^{n}} e^{-\frac{q|u|^{2}}{20}} d v(u)\right)^{\frac{1}{q}}
$$

and we finally get that

$$
\int_{\mathbb{C}^{n}}\left|K_{R}^{f}(z, u)\right| h(u) d \mu(u) \leq C_{1, R} h(z),
$$

where $C_{1, R} \rightarrow 0$ as $R \rightarrow+\infty$.

The first inequality is derived similarly by noting that

$$
\begin{aligned}
\int_{\mathbb{C}^{n}}\left|K_{R}^{f}(z, u)\right| h(z) d \mu(z) & \leq \int_{\mathbb{C}^{n}}\left|T_{\bar{f}} K_{z}(u)\right|\left(K_{z}(z)\right)^{\frac{1}{2}} d \mu(z) \\
& =(2 \pi)^{-n} \int_{\mathbb{C}^{n}}\left|K_{z}(u)\right|\left|P\left(\bar{f} \circ \tau_{z}\right)\left(\tau_{z}(u)\right)\right| e^{-\frac{|z|^{2}}{4}} d v(z) \\
& =(2 \pi)^{-n} \int_{\mathbb{C}^{n}}\left|K_{z+u}(u)\right|\left|P\left(\bar{f} \circ \tau_{z+u}\right)(z)\right| e^{-\frac{|z+u|^{2}}{4}} d v(z) \\
& =(2 \pi)^{-n} \int_{\mathbb{C}^{n}}\left|e^{\frac{u \cdot(z+u)}{2}} \| P\left(\bar{f} \circ \tau_{z+u}\right)(z)\right| e^{-\frac{|z+u|^{2}}{4}} d v(z) .
\end{aligned}
$$


However,

$$
\begin{aligned}
\left|e^{\frac{u \cdot(z+u)}{2}}\right| e^{-\frac{|z+u|^{2}}{4}} & =e^{\frac{\operatorname{Re}\{u \cdot z\}}{2}} e^{\frac{|u|^{2}}{2}} e^{-\frac{|z|^{2}}{4}} e^{-\frac{\operatorname{Re}\{u \cdot z\}}{2}} e^{-\frac{|u|^{2}}{4}} \\
& =e^{\frac{|u|^{2}}{4}} e^{-\frac{|z|^{2}}{4}}
\end{aligned}
$$

and by Step 1 , we have that $\left|P\left(\bar{f} \circ \tau_{z+u}\right)(z)\right| \leq K_{f} e^{\frac{|z|^{2}}{8}}$ (with $K_{f}$ independent of $u$ ) so that

$$
\int_{\mathbb{C}^{n}}\left|K_{R}^{f}(z, u)\right| h(z) d \mu(z) \leq(2 \pi)^{-n} K_{f} e^{\frac{|u|^{2}}{4}} \int_{\mathbb{C}^{n}} e^{-\frac{|z|^{2}}{8}} d v(z)=C_{2} h(u) .
$$

By Schur's test, this proves Step 3, and thus proves Theorem 1.

Remark. The converse of Theorem 1 is established in the next section, as is the converse of Theorem 2. Since it is not obvious that $\widetilde{T_{f}}=\tilde{f}$ for arbitrary $f$ in $\mathrm{BMO}^{1}\left(\mathbb{C}^{n}\right)$, these results are not trivial.

\section{Properties of $\mathrm{BMO}^{p}\left(\mathbb{C}^{n}\right)$}

In this short section, we state some basic properties about $\mathrm{BMO}^{p}\left(\mathbb{C}^{n}\right)$ and provide converses to Theorems 1 and 2 in the previous section.

As in $\left[\mathrm{Z}\right.$, for $1 \leq p<\infty$, we say that $f$ is in $\mathrm{BMO}^{p}\left(\mathbb{C}^{n}\right)$ if $f \in \mathcal{T}^{p}\left(\mathbb{C}^{n}\right)$ and

$$
\sup _{z \in \mathbb{C}^{n}}\left\|f \circ \tau_{z}-\tilde{f}(z)\right\|_{L^{p}\left(\mathbb{C}^{n}, d \mu\right)}<\infty .
$$

For a function $f \in \mathrm{BMO}^{p}\left(\mathbb{C}^{n}\right)$, we denote the supremum above by $\|f\|_{\mathrm{BMO}^{p}\left(\mathbb{C}^{n}\right)}$.

As $d \mu$ is a probability measure, it is not hard to see that

$$
\begin{array}{r}
L^{\infty}\left(\mathbb{C}^{n}\right) \subset \mathrm{BMO}^{p}\left(\mathbb{C}^{n}\right) \subset L^{p}\left(\mathbb{C}^{n}, d \mu\right), \quad \text { for } p \geq 1, \\
\mathrm{BMO}^{q}\left(\mathbb{C}^{n}\right) \subset \mathrm{BMO}^{p}\left(\mathbb{C}^{n}\right) \subset \mathrm{BMO}^{1}\left(\mathbb{C}^{n}\right), \quad \text { for } 1 \leq p<q,
\end{array}
$$

where each of these inclusions is strict.

Also, as in [Zh], we can give a much more detailed description of the spaces $\mathrm{BMO}^{p}\left(\mathbb{C}^{n}\right)$ for $1 \leq p<\infty$. In particular, Theorem 5 in $[\mathrm{Zh}]$ holds for $\mathrm{BMO}^{p}\left(\mathbb{C}^{n}\right)$ for $1 \leq p<\infty$ provided that we appropriately modify the spaces involved in Theorem 5 to the $\mathbb{C}^{n}$ case, where we use the weighted measures $d \mu_{t}(w)=\left(\frac{t}{\pi}\right)^{n} e^{-t|w|^{2}} d v(w)$ for $t>0$ instead of the standard weighted measures on the unit ball $\mathbb{B}_{n}$. Since the proof is very similar to the proof in [ $\mathrm{Zh}]$, we leave it to the interested reader.

This gives us that the only entire functions in $\mathrm{BMO}^{p}\left(\mathbb{C}^{n}\right)$ for $1 \leq p<\infty$ are linear polynomials. In particular, if $B(z, 1)$ is a Euclidean ball of radius 1 centered at $z$ and $f_{B(z, 1)}$ denotes the average of $f$ over the ball $B(z, 1)$, then $\mathrm{BMO}^{p}\left(\mathbb{C}^{n}\right)=$ $\mathrm{BO}\left(\mathbb{C}^{n}\right)+\mathrm{BA}^{p}\left(\mathbb{C}^{n}\right)$ via the decomposition $f=f_{B(\cdot, 1)}+\left(f-f_{B(\cdot, 1)}\right)$, where we say that $f \in \mathrm{BO}\left(\mathbb{C}^{n}\right)$ if

$$
\sup _{z \in \mathbb{C}^{n}} \omega(f)(z)<\infty
$$

with $\omega(f)(z)=\sup _{w \in B(z, 1)}|f(z)-f(w)|$ and we say $f \in \mathrm{BA}^{p}\left(\mathbb{C}^{n}\right)$ if

$$
\sup _{z \in \mathbb{C}^{n}} \frac{1}{v(B(z, 1))} \int_{B(z, 1)}|f(w)|^{p} d v(w)<\infty .
$$

Since $f$ is entire, $f=f_{B(\cdot, 1)}$ is in $\operatorname{BO}\left(\mathbb{C}^{n}\right)$, and so an easy application of the Cauchy estimates now shows that $f$ must be a linear polynomial. 
We also see that $\|\cdot\|_{\mathrm{BMO}^{p}\left(\mathbb{C}^{n}\right)}$ is a complete semi-norm on $\mathrm{BMO}^{p}\left(\mathbb{C}^{n}\right)$. If we denote the supremums in (2) and (3) by $\|\cdot\|_{\mathrm{BO}\left(\mathbb{C}^{n}\right)}$ and $\|\cdot\|_{\mathrm{BA}^{p}\left(\mathbb{C}^{n}\right)}$, respectively, then it is not difficult to show that $\|\cdot\|_{\mathrm{BA}^{p}\left(\mathbb{C}^{n}\right)}$ and $\|\cdot\|_{\mathrm{BO}\left(\mathbb{C}^{n}\right)}$ are complete semi-norms on $\mathrm{BA}^{p}\left(\mathbb{C}^{n}\right)$ and $\mathrm{BO}\left(\mathbb{C}^{n}\right)$, respectively. Moreover, a careful analysis of Theorem 5 in Zh gives us that on $\mathrm{BMO}^{p}\left(\mathbb{C}^{n}\right)=\mathrm{BO}\left(\mathbb{C}^{n}\right)+\mathrm{BA}^{p}\left(\mathbb{C}^{n}\right)$, the semi-norm $\|\cdot\|_{\mathrm{BMO}^{p}\left(\mathbb{C}^{n}\right)}$ is equivalent to the semi-norm

$$
\begin{aligned}
\|f\|_{\mathrm{BA}^{p}\left(\mathbb{C}^{n}\right)+\mathrm{BO}\left(\mathbb{C}^{n}\right)=\inf \left\{\left\|f_{1}\right\|_{\mathrm{BA}^{p}\left(\mathbb{C}^{n}\right)}\right.}+\left\|f_{2}\right\|_{\mathrm{BO}\left(\mathbb{C}^{n}\right)}: f=f_{1}+f_{2} \\
\text { with } \left.f_{1} \in \mathrm{BA}^{p}\left(\mathbb{C}^{n}\right) \text { and } f_{2} \in \mathrm{BO}\left(\mathbb{C}^{n}\right)\right\} .
\end{aligned}
$$

Using the decomposition $\mathrm{BMO}^{1}\left(\mathbb{C}^{n}\right)=\mathrm{BA}^{1}\left(\mathbb{C}^{n}\right)+\mathrm{BO}\left(\mathbb{C}^{n}\right)$ and again considering the integral operator $T_{f}=P M_{f} P$ with $f \in \mathrm{BMO}^{1}\left(\mathbb{C}^{n}\right)$, we can provide the converse to Theorem 2.

Theorem 3. For $f \in B M O^{1}\left(\mathbb{C}^{n}\right)$ and $T_{f}$ bounded on $L^{2}$, we have $\widetilde{T_{f}}=\tilde{f}$ so that $\tilde{f}$ must be bounded.

Proof. We will show that

$$
\left\langle T_{f} K_{b}, K_{a}\right\rangle=\int_{\mathbb{C}^{n}} f(z) K_{b}(z) \overline{K_{a}(z)} d \mu(z)
$$

A simple application of the Cauchy-Schwarz inequality completes the proof.

By definition,

$$
\begin{aligned}
\left\langle T_{f} K_{b}, K_{a}\right\rangle & =\int_{\mathbb{C}^{n}}\left(T_{f} K_{b}\right)(u) \overline{K_{a}(u)} d \mu(u) \\
& =\int_{\mathbb{C}^{n}}\left(\int_{\mathbb{C}^{n}} f(w) K_{b}(w) e^{\frac{u \cdot w}{2}} d \mu(w)\right) \overline{K_{a}(u)} d \mu(u) .
\end{aligned}
$$

The desired result follows by interchanging the order of integration. For Fubini's theorem to hold, we need to check that

$$
\int_{\mathbb{C}^{n}}\left(\int_{\mathbb{C}^{n}}\left|f(w) e^{\frac{w \cdot b}{2}} e^{\frac{u \cdot w}{2}} e^{\frac{u \cdot a}{2}}\right| d \mu(u)\right) d \mu(w)<\infty
$$

Recall that $\mathrm{BMO}^{1}\left(\mathbb{C}^{n}\right)=\mathrm{BA}^{1}\left(\mathbb{C}^{n}\right)+\mathrm{BO}\left(\mathbb{C}^{n}\right)$, so first assume that $f \in \mathrm{BA}^{1}\left(\mathbb{C}^{n}\right)$. Treat $\mathbb{Z}^{2 n}$ as a lattice in $\mathbb{C}^{n}$ and pick an $R>0$ where $\bigcup_{\nu \in \mathbb{Z}^{2 n}} B(\nu, R)=\mathbb{C}^{n}$. Since $f \in \mathrm{BA}^{1}\left(\mathbb{C}^{n}\right)$, it is easy to see that

$$
\sup _{z \in \mathbb{C}^{n}} \int_{B(z, R)}|f(w)| d v(w)<\infty .
$$

Let $C_{f}$ denote the supremum above. Since

$$
(2 \pi)^{-n} \int_{\mathbb{C}^{n}}\left|e^{\frac{u \cdot(w+a)}{2}}\right| e^{-\frac{|u|^{2}}{2}} d v(u)=e^{\frac{|w+a|^{2}}{8}}
$$


we have that

$$
\begin{aligned}
\int_{\mathbb{C}^{n}} & \left(\int_{\mathbb{C}^{n}}\left|f(w) e^{\frac{w \cdot b}{2}} e^{\frac{u \cdot w}{2}} e^{\frac{u \cdot a}{2}}\right| d \mu(u)\right) d \mu(w) \\
& =\int_{\mathbb{C}^{n}}\left(\int_{\mathbb{C}^{n}} e^{\frac{u \cdot(w+a)}{2}} \mid d \mu(u)\right)\left|f(w) e^{\frac{w \cdot b}{2}}\right| d \mu(w) \\
& =(2 \pi)^{-n} \int_{\mathbb{C}^{n}} e^{\frac{|w+a|^{2}}{8}}\left|f(w) e^{\frac{w \cdot b}{2}}\right| e^{-\frac{|w|^{2}}{2}} d v(w) \\
& \leq(2 \pi)^{-n} \int_{\mathbb{C}^{n}} e^{\frac{(|w|+|a|)^{2}}{8}}|f(w)| e^{\frac{|w||b|}{2}} e^{-\frac{|w|^{2}}{2}} d v(w) \\
& \leq(2 \pi)^{-n} \sum_{\nu \in \mathbb{Z}^{2 n}} \int_{B(\nu, R)} e^{\frac{(|w|+|a|)^{2}}{8}}|f(w)| e^{\frac{|w||b|}{2}} e^{-\frac{|w|^{2}}{2}} d v(w) .
\end{aligned}
$$

However, for $w \in B(\nu, R)$, the triangle inequality gives us that

$$
|\nu|-R \leq|w| \leq|\nu|+R .
$$

Thus, for some constant $C_{f, a, b}$, chosen to compensate for the finitely many $\nu$ with $|\nu|<R$, we have

$$
\begin{aligned}
\int_{\mathbb{C}^{n}}\left(\int_{\mathbb{C}^{n}}\left|f(w) e^{\frac{w \cdot b}{2}} e^{\frac{u \cdot w}{2}} e^{\frac{u \cdot a}{2}}\right| d \mu(u)\right) d \mu(w) \\
\leq(2 \pi)^{-n} C_{f, a, b} \sum_{\nu \in \mathbb{Z}^{2 n}} e^{\frac{(|\nu|+R+|a|)^{2}}{8}} e^{\frac{(|\nu|+R)|b|}{2}} e^{-\frac{(|\nu|-R)^{2}}{2}} \\
<+\infty
\end{aligned}
$$

since

$$
\begin{aligned}
e^{\frac{(|\nu|+R+|a|)^{2}}{8}} e^{\frac{(|\nu|+R)|b|}{2}} e^{-\frac{(|\nu|-R)^{2}}{2}} & =e^{\frac{|\nu|^{2}}{8}} e^{\frac{2(R+|a|)|\nu|+(R+|a|)^{2}}{8}} e^{\frac{(|\nu|+R)|b|}{2}} e^{-\frac{|\nu|^{2}}{2}} e^{R|\nu|-\frac{R^{2}}{2}} \\
& \leq C_{a, b} e^{-\frac{|\nu|^{2}}{4}}
\end{aligned}
$$

for some $C_{a, b}>0$.

The case where $f \in \mathrm{BO}\left(\mathbb{C}^{n}\right)$ is even easier. Recall that $f \in \mathrm{BO}\left(\mathbb{C}^{n}\right)$ if

$$
\sup _{z \in \mathbb{C}^{n}} \omega(f)(z)<\infty
$$

with $\omega(f)(z)=\sup _{w \in B(z, 1)}|f(z)-f(w)|$. By the argument of [BBCZ, p. 329], there is a constant $A_{f}$ with $|f(w)| \leq A_{f}(1+|w|)$ for all $w$ in $\mathbb{C}^{n}$. A direct calculation shows that (4) is finite.

As a consequence of Theorem 3, we have the converse to Theorem 1.

Theorem 4. For $f \in B M O^{1}\left(\mathbb{C}^{n}\right)$, if $T_{f}$ is compact, then $\lim _{|z| \rightarrow+\infty} \tilde{f}(z)=0$.

Proof. By hypothesis, $T_{f}$ must be bounded and by Theorem $3, \tilde{f}$ is bounded with $\left\langle T_{f} k_{a}, k_{a}\right\rangle=\tilde{f}(a)$. Since $k_{a} \rightarrow 0$ weakly as $|a| \rightarrow+\infty$, the compactness of $T_{f}$ forces $\lim _{|a| \rightarrow+\infty}\left\|T_{f} k_{a}\right\|=0$ and so, using the Cauchy-Schwarz inequality, $\lim _{|a| \rightarrow+\infty} \tilde{f}(a)=0$.

Remark. By a computation that is similar but slightly more involved than the proof of Theorem 3, we can show that $T_{f}$ is densely defined on $L^{2}$ for $f \in \mathrm{BMO}^{1}\left(\mathbb{C}^{n}\right)$. 
In particular, by Fubini's theorem, we will have

$$
\left\|T_{f} K_{b}\right\|_{L^{2}}^{2}=\int_{\mathbb{C}^{n}}\left|\int_{\mathbb{C}^{n}} f(w) e^{\frac{w \cdot b}{2}} e^{\frac{z \cdot w}{2}} d \mu(w)\right|^{2} d \mu(z)<\infty
$$

for all $b \in \mathbb{C}^{n}$ if

$$
\begin{aligned}
& \int_{\mathbb{C}^{n}}\left(\int_{\mathbb{C}^{n}}\left(\int_{\mathbb{C}^{n}}\left|e^{\frac{z \cdot(u+w)}{2}}\right| d \mu(z)\right)|f(w)||f(u)| e^{\frac{|b||u+w|}{2}} d \mu(u)\right) d \mu(w) \\
& =\int_{\mathbb{C}^{n}}\left(\int_{\mathbb{C}^{n}}|f(w)||f(u)| e^{\frac{|u+w|^{2}}{8}} e^{\frac{|b||u+w|}{2}} d \mu(u)\right) d \mu(w)<\infty .
\end{aligned}
$$

But picking $R>0$ where $\bigcup_{\nu \in \mathbb{Z}^{2 n}} B(\nu, R)=\mathbb{C}^{n}$ and using the decomposition $\mathrm{BMO}^{1}\left(\mathbb{C}^{n}\right)=\mathrm{BA}^{1}\left(\mathbb{C}^{n}\right)+\mathrm{BO}\left(\mathbb{C}^{n}\right)$, we have that (5) is finite for $f \in \mathrm{BMO}^{1}\left(\mathbb{C}^{n}\right)$. Moreover, the proof of Theorem 3 now tells us that $\widetilde{T_{f}}=\tilde{f}$ and that $T_{f}$ maps the dense linear span of $\left\{K_{z}: z \in \mathbb{C}^{n}\right\}+\left(H^{2}\right)^{\perp}$ in $L^{2}$ into $H^{2}$ if $f \in \mathrm{BMO}^{1}\left(\mathbb{C}^{n}\right)$.

Also, using the fact that

$$
\left\langle T_{f} K_{b}, K_{a}\right\rangle=\int_{\mathbb{C}^{n}} f(z) K_{b}(z) \overline{K_{a}(z)} d \mu(z)
$$

for all $f \in \mathrm{BMO}^{1}\left(\mathbb{C}^{n}\right)$, we can check that the map $f \mapsto T_{f}$ is injective on $\mathrm{BMO}^{1}\left(\mathbb{C}^{n}\right)$. In particular, since $f \in \mathrm{BMO}^{1}\left(\mathbb{C}^{n}\right)$, we have that $g(z)=f(z) e^{-\frac{|z|^{2}}{2}}$ is in $L^{1}\left(\mathbb{C}^{n}, d v\right)$. By taking $b=-a$, it is not difficult to check that the Fourier transform $\hat{g} \equiv 0$ if $T_{f}=0$. It follows that $g=0$ a.e. and so $f=0$ a.e.

Now, if $p>1$, then we have that $\tilde{f}$ is Lipschitz if $f \in \mathrm{BMO}^{p}\left(\mathbb{C}^{n}\right)$. More generally, we have

Theorem 5. Let $\gamma: I \equiv[0,1] \rightarrow \mathbb{C}^{n}$ be a smooth curve and $f \in B M O^{p}\left(\mathbb{C}^{n}\right)$. Then for any $t \in I$, we have

$$
\left|\frac{d}{d t} \tilde{f}(\gamma(t))\right| \leq 2 C_{p}\|f\|_{B M O^{p}(\gamma(I))}\left|\frac{d}{d t} \gamma(t)\right|,
$$

where $C_{p}$ only depends on $p$ and where

$$
\|f\|_{B M O^{p}(\gamma(I))}=\sup _{t \in I}\left\|f \circ \tau_{\gamma(t)}-\tilde{f}(\gamma(t))\right\|_{L^{p}\left(\mathbb{C}^{n}, d \mu\right)} .
$$

Proof. According to [Bau, p. 6], we have that

$\left|\frac{d}{d t} \tilde{f}(\gamma(t))\right|=\left.2\left|\int_{\mathbb{C}^{n}}(f(u)-\tilde{f}(\gamma(t))) \operatorname{Re}\left((u-\gamma(t)) \cdot \frac{d}{d t} \gamma(t)\right)\right| k_{\gamma(t)}(u)\right|^{2} d \mu(u) \mid$.

If $t \in I$ is fixed, then the result now holds by applying Hölder's inequality to the previous equation with respect to the measure $\left|k_{\gamma(t)}(u)\right|^{2} d \mu(u)$.

Note that this was proven for $p=2$ in $\mathrm{Bau}$ with constant $C_{2}=1$.

It is obvious that the above theorem implies that $\tilde{f}$ is Lipschitz if $f \in \mathrm{BMO}^{p}\left(\mathbb{C}^{n}\right)$ for $1<p<\infty$. Recall that the corollary to Theorem 2 states that $\tilde{f}$ is Lipschitz if $f$ is in $\mathrm{BMO}^{1}\left(\mathbb{C}^{n}\right)$ and $\tilde{f}$ is bounded. We do not know if Theorem 5 holds for $p=1$ or whether the weaker conclusion that $\tilde{f}$ is Lipschitz when $f \in \mathrm{BMO}^{1}\left(\mathbb{C}^{n}\right)$ is true. 
Remark. As mentioned in Remark 1 after Theorem 2, we can show, at least, that $\tilde{f}$ is continuous for all $f \in \mathrm{BMO}^{1}\left(\mathbb{C}^{n}\right)$. This can be seen as follows: if $f \in \operatorname{BO}\left(\mathbb{C}^{n}\right)$, then clearly there is some $C>0$ where

$$
|f(z)| \leq C(1+|z|)
$$

for all $z \in \mathbb{C}^{n}$. Thus, for $f \in \mathrm{BO}\left(\mathbb{C}^{n}\right)$, the continuity of $\tilde{f}$ follows from an easy application of the Lebesgue dominated convergence theorem. If $f \in \mathrm{BA}^{1}\left(\mathbb{C}^{n}\right)$, then another computation that is similar to the proof of Theorem 3 gives us that $\widetilde{|f|}$ is bounded. It now follows from Theorem 2 and [C] that $\tilde{f}$ is continuous (and in fact Lipschitz) since $T_{f}$ is bounded on $H^{2}\left(\mathbb{C}^{n}, d \mu\right)$.

\section{COMPaCTNESS OF Windowed Fourier LOCALiZATION OPERATORS}

We now give a criterion for the compactness of windowed Fourier localization operators $L_{f}^{(w)}$ where the window $w$ is a linear combination of Hermite functions and $f$ is in a natural $\mathrm{BMO}^{1}\left(\mathbb{C}^{n}\right)$ Sobolev-type space. Since the Bargmann isome$\operatorname{try} \beta$ is surjective and maps linear combinations of Hermite functions to analytic polynomials, it will be enough to give a compactness criterion for the operator $\beta L_{f}^{(w)} \beta^{-1}$ on $H^{2}\left(\mathbb{C}^{n}, d \mu\right)$.

Following [E2, let $k(z)=(2 \pi)^{-n}|\beta w(z)|^{2} e^{-\frac{|z|^{2}}{2}}$ and $h(z)=(2 \pi)^{-n} e^{-\frac{|z|^{2}}{2}}$, and let $D=D^{(w)}$ be the unique linear constant coefficient partial differential operator of order $2 d(\beta w)$, where $D h=k$ and $d(\beta w)$ is the degree of $\beta w$. Explicitly, the operator $D$ was given in [E2] by

$$
D^{(w)}=\left[e^{\frac{\Delta}{2}}|\beta w(z)|^{2}\right]_{\substack{z \mapsto-2 \bar{\partial} \\ \bar{z} \mapsto-2 \partial}} \cdot
$$

It was also shown in E2] that $T_{D f}=\beta L_{f}^{(w)} \beta^{-1}$ holds for any function $f$ that is weakly differentiable up to order $2 d(\beta w)$ and satisfies

$$
h * D f=D h * f=k * f
$$

(where it is assumed that $f$ is 'nice' enough for the convolution product to exist). Thus, for a fixed linear combination $w$ of Hermite functions, we describe a natural function space (depending on $w$ ) of symbols that will ensure that (7) holds.

For some $m \in \mathbb{N}$ and $1 \leq p<\infty$, let $W^{m, p}\left(\mathbb{C}^{n}, d \mu\right)$ denote the weighted Sobolev space with Gaussian weight $d \mu$ consisting of weakly differentiable functions up to order $m$ whose derivatives are in $L^{p}\left(\mathbb{C}^{n}, d \mu\right)$, and give $W^{m, p}\left(\mathbb{C}^{n}, d \mu\right)$ the standard norm

$$
\|f\|_{W^{m, p}\left(\mathbb{C}^{n}, d \mu\right)}=\sum_{0 \leq|\alpha| \leq m}\left\|D^{\alpha} f\right\|_{L^{p}\left(\mathbb{C}^{n}, d \mu\right)}
$$

If we set $d \mu_{w}(z)=|\beta w(z)|^{2} e^{-\frac{|z|^{2}}{2}} d v(z)$, then define $\mathcal{T}_{w}^{m, p}\left(\mathbb{C}^{n}\right)$ to be the set of all $f$ such that for each $z \in \mathbb{C}^{n}$, the function $f \circ \tau_{z}$ is in the closure of $C_{c}^{m}\left(\mathbb{C}^{n}\right)$ in $L^{p}\left(\mathbb{C}^{n}, d \mu_{w}\right) \cap W^{m, p}\left(\mathbb{C}^{n}, d \mu\right)$. Since $C_{c}\left(\mathbb{C}^{n}\right)$ is dense in $L^{p}\left(\mathbb{C}^{n}, d \mu\right), \mathcal{T}_{w}^{m, p}\left(\mathbb{C}^{n}\right)=$ $\mathcal{T}^{p}\left(\mathbb{C}^{n}\right)$ when $\beta w \equiv 1$ and $m=0$. Thus, the spaces $\mathcal{T}_{w}^{m, p}\left(\mathbb{C}^{n}\right)$ are regularized generalizations of $\mathcal{T}^{p}\left(\mathbb{C}^{n}\right)$. Also, for $r>0$, if we let $M_{r}$ denote the space of functions (introduced in [E2]) given by

$M_{r}=\left\{f \in C^{2 r}\left(\mathbb{C}^{n}\right)\right.$ : for any $a>0$ and $\left.|j|,|k| \leq r, e^{a|\cdot|}\left|\partial^{j} \bar{\partial}^{k} f\right| e^{-\frac{|\cdot|^{2}}{2}} \in L^{\infty}\left(\mathbb{C}^{n}\right)\right\}$, 
then we have $M_{d(\beta w)} \subset \mathcal{T}_{w}^{2 d(\beta w), 1}\left(\mathbb{C}^{n}\right)$. In particular, if $f \in M_{d(\beta w)}$ and if $h \in$ $C_{c}^{\infty}\left(\mathbb{C}^{n}\right)$, where $h: \mathbb{C}^{n} \rightarrow[0,1]$ and $h \equiv 1$ on $B(0,1)$, then it is not hard to see that $f_{\ell} \rightarrow f$ in $L^{p}\left(\mathbb{C}^{n}, d \mu_{w}\right) \cap W^{m, p}\left(\mathbb{C}^{n}, d \mu\right)$, where $f_{\ell}(z)=h\left(\frac{z}{\ell}\right) f(z)$.

For $f \in \mathcal{T}_{w}^{2 d(\beta w), 1}\left(\mathbb{C}^{n}\right)$, a simple density argument shows that (7) holds. Therefore, if we say that $f \in \mathrm{BMO}_{w}^{m, p}\left(\mathbb{C}^{n}\right)$ when $f \in \mathcal{T}_{w}^{m, p}\left(\mathbb{C}^{n}\right)$ and all weak derivatives of $f$ up to order $m$ are in $\mathrm{BMO}^{p}\left(\mathbb{C}^{n}\right)$, then we have the following.

Theorem 6. For a linear combination $w$ of Hermite functions, let $D=D^{(w)}$ be the differential operator given by (6) and let $f \in B M O_{w}^{2 d(\beta w), 1}\left(\mathbb{C}^{n}\right)$. Then $L_{f}^{(w)}$ is compact if and only if $\lim _{|z| \rightarrow+\infty} \widetilde{D f}(z)=0$.

Proof. Note that if $f \in \mathcal{T}_{w}^{m, p}\left(\mathbb{C}^{n}\right)$, then the weak derivatives of $f$ up to order $m$ are in $\mathcal{T}^{p}\left(\mathbb{C}^{n}\right)$. Thus, the result follows immediately from Theorem 1 and the discussion above.

\section{EXAmPles AND COUNTEREXAMPles}

As discussed earlier, $L^{p}\left(\mathbb{C}^{n}, d v\right)$ for $1 \leq p<\infty$ provides an interesting application of Theorem 1. We have

Theorem 7. For $f$ in some $L^{p}\left(\mathbb{C}^{n}, d v\right)$ with $1 \leq p<\infty, f$ is in $B M O^{1}\left(\mathbb{C}^{n}\right)$ with $\lim _{|a| \rightarrow+\infty} \widetilde{|f|}(a)=0$.

Proof. This is a " $2 \epsilon$-argument", using Hölder's inequality. We split the integral for $\widetilde{|f|}(a)$ into integrals outside and inside the open ball centered at 0 with radius $R$. For $\frac{1}{p}+\frac{1}{q}=1$, we get

$$
(2 \pi)^{-n} \int_{|z| \geq R}|f(z)| e^{-\frac{|z-a|^{2}}{2}} d v(z) \leq C_{q}\left\{\int_{|z| \geq R}|f(z)|^{p} d v(z)\right\}^{\frac{1}{p}}
$$

and, for any given $\epsilon>0$, we can take $R$ large enough to get

$$
C_{q}\left\{\int_{|z| \geq R}|f(z)|^{p} d v(z)\right\}^{\frac{1}{p}}<\frac{\epsilon}{2} .
$$

For this $R$, we note that $f \in L^{1}(B(0, R), d v)$ and, for $|a| \geq R$,

$$
e^{-\frac{(|a|-R)^{2}}{2}} \geq e^{-\frac{|a-z|^{2}}{2}} .
$$

Taking $|a|$ sufficiently large completes the proof.

Remark 1. By Theorem 1 , for $f$ in some $L^{p}\left(\mathbb{C}^{n}, d v\right)$ with $1 \leq p<\infty, T_{f}$ is compact. For $f$ in $L^{1}\left(\mathbb{C}^{n}, d v\right)$, it is "well known" [F, p. 142], Be1] that $T_{f}$ is trace class with

$$
\operatorname{trace}\left(T_{f}\right)=(2 \pi)^{-n} \int_{\mathbb{C}^{n}} f(z) d v(z) .
$$

A rigorous proof of this fact can be given using Remark 1 after Theorem 2 and standard properties of Bergman spaces.

Remark 2. Let $W^{m, p}\left(\mathbb{C}^{n}, d v\right)$ be the Sobolev space of weakly differentiable functions whose derivatives up to order $m$ are in $L^{p}\left(\mathbb{C}^{n}, d v\right)$ for $1 \leq p<\infty$. It is easy to check that for $f$ in $W^{m, p}\left(\mathbb{C}^{n}, d v\right), f \circ \tau_{a}$ is also in $W^{m, p}\left(\mathbb{C}^{n}, d v\right)$ for all $a$ in $\mathbb{C}^{n}$. Moreover, $C_{c}^{m}\left(\mathbb{C}^{n}\right)$, the set of compactly supported $m$-times differentiable functions, is dense 
in $W^{m, p}\left(\mathbb{C}^{n}\right)$ [AF, p. 67]. We can now check that $W^{m, p}\left(\mathbb{C}^{n}, d v\right)$ is embedded in $\mathcal{T}_{w}^{m, 1}\left(\mathbb{C}^{n}\right)$ by standard use of Hölder's inequality. Theorem 7 shows that for $f$ in $W^{m, p}\left(\mathbb{C}^{n}, d v\right), f$ is in $\mathrm{BMO}_{w}^{m, 1}\left(\mathbb{C}^{n}\right)$. Moreover, $\widetilde{\left|D^{\alpha} f\right|}(a)$ vanishes at infinity for all $|\alpha| \leq m$. Taking $m=2 d(\beta w)$, we see that $\lim _{|z| \rightarrow+\infty} \widetilde{D f}(z)=0$, where $D$ is the differential operator given by (6), and by Theorem 6 , the windowed Fourier localization operator $L_{f}^{(w)}$ is compact.

As noted in the introduction, [BC2, p. 582] provides counterexamples to the unrestricted applicability of the compactness criterion in Theorem 1. In particular, on $H^{2}(\mathbb{C}, d \mu)$, we can take

$$
f(z)=e^{\left(\frac{1}{5}+i \frac{2}{5}\right)|z|^{2}} .
$$

Then $f$ is in $\mathcal{T}(\mathbb{C})$ and $T_{f}$ is unitary (and thus noncompact) while

$$
\tilde{f}(z)=\left(\frac{3}{5}+i \frac{4}{5}\right) e^{\left(-\frac{1}{5}+i \frac{2}{5}\right)|z|^{2}} .
$$

By direct calculations, $f$ is not in $\operatorname{BMO}^{1}(\mathbb{C})$.

\section{REFERENCES}

[AF] R. Adams and J. Fournier, Sobolev Spaces, Academic Press, 2nd ed., 2003. MR2424078 (2009e:46025)

[AZ] S. Axler and D. Zheng, Compact operators via the Berezin transform, Indiana Univ. Math. J., 47(1998), 387-400. MR1647896(99i:47045)

[Ba] V. Bargmann, On a Hilbert space of analytic functions and an associated integral transform, Communications on Pure and Applied Mathematics, 14 (1961), 187-214. MR0157250 (28:486)

[Bau] W. Bauer, Mean oscillation and Hankel operators on the Segal-Bargmann space, Integral Equations and Operator Theory, 52 (2005) 1-15. MR2138695 (2006b:47033)

[BBCZ] D. Bèkollè, C. A. Berger, L. A. Coburn, and K. Zhu, BMO in the Bergman metric on bounded symmetric domains, J. Funct. Anal., 93 (1990), 310-350. MR1073289 $(91 \mathrm{j}: 32034)$

[BC1] C. A. Berger and L. A. Coburn, Toeplitz operators on the Segal-Bargmann space, Transactions Amer. Math. Soc., 301 (1987), 813-829. MR882716 (88c:47044)

[BC2] C. A. Berger and L. A. Coburn, Heat flow and Berezin-Toeplitz estimates, American Journal of Mathematics, 116 (1994), 563-590. MR1277446 (95g:47038)

[Be1] F. A. Berezin, Wick and anti-Wick operator symbols, Math. USSR Sb., 86(128) (1971), 578-610. MR 0291839 (45:929)

$\left[\mathrm{Be}_{2}\right] \quad$ F. A. Berezin, Covariant and contravariant symbols of operators, Math. USSR Izv., 6 (1972), 1117-1151. MR0350504 (50:2996)

$[\mathrm{BF}]$ W. Bauer and K. Furutani, Compact operators and the pluriharmonic Berezin transform, Internat. J. Math., 19 (2008), 645-669. MR2431632(2010b:47070)

[C] L. A. Coburn, A Lipschitz estimate for Berezin's operator calculus, Proceedings of the American Mathematical Society, 133 (2005), no. 1, 127-131. MR2085161|(2005e:47060)

[D] I. Daubechies, Time-frequency localization operators: A geometric phase space approach, IEEE Trans. Inform. Theory, 34 (1988), 605-612. MR966733

[E1] M. Engliš, Compact Toeplitz operators via the Berezin transform on bounded symmetric domains, Integral Equations and Operator Theory, 33 (1999), 426-455. MR1682815 (2000h:47050a)

[E2] M. Engliš, Toeplitz operators and localization operators, Transactions of the American Mathematical Society, 361 (2009), 1039-1052. MR2452833(2010a:47056)

[F] G. B. Folland, Harmonic analysis in phase space, Annals of Mathematical Studies 122, Princeton University Press, 1989. MR $983366(92 \mathrm{k}: 22017)$

[HS] P. Halmos and V. Sunder, Bounded integral operators on $L^{2}$ spaces, Springer Verlag, 1978. MR517709 (80g:47036) 
[K] S. Krantz, Function theory of several complex variables, Amer. Math. Soc., Providence, RI, 2nd ed., 2001. MR1846625 (2002e:32001)

[L] M. Lo, The Bargmann transform and windowed Fourier localization, Integral Equations and Operator Theory, 57 (2007), 397-412. MR2307818(2008b:47049)

[MZ] J. Miao and D. Zheng, Compact operators on Bergman Spaces, Integral Equations and Operator Theory, 48 (2004), 61-79. MR2029944 (2004k:47056)

[RN] F. Riesz and B. Nagy. Functional analysis, Dover Publications, 1990. MR.1068530 (91g:00002)

[Z] N. Zorboska, Toeplitz operators with BMO symbols and the Berezin transform, Int. J. Math and Math. Sci., 46 (2003), 2929-2945. MR2007108 (2004g:47039)

[Zh] K. Zhu, BMO and Hankel operators on Bergman spaces, Pacific Jour. of Math., 155 (1992), 377-397. MR1178032 (93g:47033)

Department of Mathematics, SUnY at Buffalo, Buffalo, New York 14260

E-mail address: lcoburn@buffalo.edu

Department of Mathematics, Suny at Buffalo, Buffalo, New York 14260

E-mail address: jbi2@buffalo.edu

Current address: Institute of Mathematics, University of Göttingen, Bunsenstrasse 3-5, D-37073 Göttingen, Germany

Department of Mathematics, SUNy at Buffalo, Buffalo, New York 14260

E-mail address: boli@buffalo.edu

Current address: Department of Mathematics and Statistics, Bowling Green State University, Bowling Green, Ohio 43403

E-mail address: boli@bgsu.edu 